Research Paper

\title{
CircMETTL3, upregulated in a m6A-dependent manner, promotes breast cancer progression
}

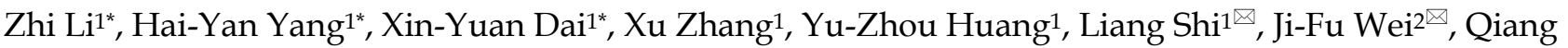 \\ Ding $1^{\bowtie}$ \\ 1. Jiangsu Breast Disease Center, the First Affiliated Hospital with Nanjing Medical University, 300 Guangzhou Road, Nanjing 210029, Jiangsu Province, P.R. China. \\ 2. Research Division of Clinical Pharmacology, the First Affiliated Hospital with Nanjing Medical University, 300 Guangzhou Road, Nanjing 210029, Jiangsu Province, \\ P.R. China. \\ *These authors equally contributed to the work.
}

$\triangle$ Corresponding authors: Qiang Ding, Jiangsu Breast Disease Center, The First Affiliated Hospital with Nanjing Medical University, 300 Guangzhou Road, Nanjing 210029, Jiangsu Province, P.R. China. E-mail: dingqiang@njmu.edu.cn; Ji-Fu Wei, Research Division of Clinical Pharmacology, the First Affiliated Hospital with Nanjing Medical University, 300 Guangzhou Road, Nanjing 210029, Jiangsu Province, P.R. China. E-mail: weijifu@hotmail.com; Liang Shi, Jiangsu Breast Disease Center, the First Affiliated Hospital with Nanjing Medical University, 300 Guangzhou Road, Nanjing 210029, Jiangsu Province, P.R. China. E-mail: shiliang@njmu.edu.cn.

(C) The author(s). This is an open access article distributed under the terms of the Creative Commons Attribution License (https://creativecommons.org/licenses/by/4.0/). See http://ivyspring.com/terms for full terms and conditions.

Received: 2021.01.03; Accepted: 2021.03.04; Published: 2021.03.15

\begin{abstract}
Growing evidence indicates N6-methyladenosine (m6A) has biological function in oncogenesis. METTL3, the catalytic component, is the most important part of methyltransferase complex and plays a crucial role in cancers. However, the biological function of circRNAs derived from METTL3 in breast cancer and the underlying molecular mechanism remains unclear. Herein, we report circMETTL3, which has not been explored in breast cancer, and it is markedly upregulated in breast cancer. Moreover, we uncovered that circMETTL3 could facilitate cell proliferation, migration and invasion in breast cancer. Mechanism investigation showed that circMETTL3 might act as a competing endogenous RNA (ceRNA) of miR-31-5p and upregulate its target cyclin-dependent kinases (CDK1). Moreover, m6A modification of circMETTL3 might affect its expression. Taken together, our results elucidate that circMETTL3 promotes breast cancer progression through circMETTL3/miR-31-5p/CDK1 axis. Moreover, METTL3, the host gene of circMETTL3, may regulate circMETTL3 expression in an m6A-dependent manner, while circMETTL3 has no effect on METTL3 expression, providing a new relationship between the circRNA and the corresponding host gene. Thus, it may serve as a new therapeutic target for breast cancer.
\end{abstract}

Key words: circMETTL3, miR-31-5p, CDK1, N6-methyladenosine, breast cancer

\section{Introduction}

Breast cancer is the most commonly diagnosed malignancy and most frequent cause of cancer-related mortality in women [1,2]. Despite the outstanding advances in the diagnosis and treatment of breast cancer in recent decades, the morbidity and mortality are still high, due to high frequency of metastasis, recurrence, and drug resistance. Therefore, it is necessary to further study the molecular mechanism and develop more efficient and precise targeting therapies to treat breast cancer.

Circular RNAs (circRNAs) are a subgroup of non-coding RNAs and covalently closed loops formed by back-splicing. They are highly conserved and stable due to the lack of 5'-cup structure and 3'-polyadenylated tail [3-5]. It has been demonstrated that
circRNAs are produced by a non-canonical alternative splicing, and RNA-binding proteins or transcription factor facilitate the generation of circRNAs $[6,7]$. Growing studies showed that circRNAs played a critical role in various biological processes in cancers, including proliferation, metastasis and autophagy, via acting as a sponge of miRNAs, interacting with RNA binding proteins or encoding peptides [8-10]. In breast cancer, many circRNAs were found to play an important role in tumor progression [11-13]. For example, circFBXW7 inhibited breast cancer by sponging miR-197-3p and encoding a 185-aa protein [13]. circSMARCA5 inhibited DNA damage repair by interacting with host gene [14]. 
N6-methyladenosine (m6A) methylation, one of the most prevalent modifications in RNA, affects RNA splicing, stability, translation $[15,16]$. m6A modification is a dynamic process, controlled by methyltransferase enzymes (including METTL3, METTL14, WTAP, KIAA1429 et al.) and demethylase enzymes (ALKBH5 and FTO) [17]. Previous studies showed these proteins played important roles in breast cancer developments [18-22], especially METTL3, which is the key component in methyltransferase enzymes [20, 23-25]. For example, METTL3 could promote breast cancer progression via inhibiting tumor suppressor let-7g or targeting Bcl-2 [20, 25], though another study showed METTL3 inhibited metastasis of triple-negative breast cancer by decreasing COL3A1 [24]. METTL3 also increased adenylate kinase 4 expression, causing the resistance of breast cancer cells to tamoxifen [23]. A recent interesting study displayed circ_KIAA1429 derived from KIAA1429, a part of methyltransferase complex, could accelerate hepatocellular carcinoma advancement through the mechanism of m6A-YTHDF3-Zeb1 [26]. We then analyzed the expression profiles of circRNAs in breast cancer tissues by analyzing GEO database (GSE113230) and characterized a novel circRNA derived from the METTL3 gene, termed as circMETTL3. This circRNA is a $623 \mathrm{nt}$ transcript originated from the exon-2 and exon-3 of METTL3 gene. However, the function of circMETTL3 in breast cancer is still unknown.

In this study, we found circMETTL3 was upregulated in breast cancer tissues and cell lines in an m6A-dependent manner. It could promote breast cancer proliferation and metastasis. Mechanistically, circMETTL3 sponged miR-31-5p to upregulate CKD1 expression. Our findings suggested that circMETTL3 might serve as a potential biomarker and therapeutic target for breast cancer patients.

\section{Materials and Methods}

\section{Clinical samples}

Breast cancer tissues and adjacent normal tissues were obtained from the First Affiliated Hospital with Nanjing Medical University. All patients received no neoadjuvant therapy. The collected samples were frozen in liquid nitrogen immediately after resection. All patients provided written informed consent, and the study was approved by the Ethics Committee of the First Affiliated Hospital with Nanjing Medical University.

\section{Cell culture}

Human breast cancer cell lines (MCF-7, ZR-75-1, BT474, MDA-MB-231, MDA-MB-453 and HCC1806) and normal mammary epithelial cell line (MCF-10A) were purchased from American Type Culture Collection (ATCC) (Manassas, VA, USA). SUM1315 was kindly provided by Stephen Ethier (University of Michigan, AnnArbor, MI, USA). 293T cell line was preserved by our lab. MCF-7, ZR-75-1, BT474, MDA-MB-231, MDA-MB-453, SUM1315 and 293T cells were cultured in DMEM (Gibco, USA), HCC1806 cells were cultured with RPMI 1640 (Gibco, USA), containing $10 \%$ fetal bovine serum, $100 \mathrm{U} / \mathrm{mL}$ penicillin and $100 \mathrm{mg} / \mathrm{mL}$ streptomycin. Cells in the medium were incubated in a humidified atmosphere containing $5 \% \mathrm{CO}_{2}$ at $37^{\circ} \mathrm{C}$.

\section{RNA extraction and quantitative real time polymerase chain reaction (qRT-PCR)}

Total RNA from cells or tissues was isolated using Trizol reagent (Takara, Japan). Complementary DNA (cDNA) was synthesized using the HiScript Q RT SuperMix (Vazyme, China). Then, qRT-PCR was performed using AceQ qPCR SYBR Green Master Mix (Vazyme, China). For miRNA expression analysis, cDNA was specifically synthesized and miRNA was detected with Bulge-Loop ${ }^{\mathrm{TM}}$ miRNA qRT-PCR (Ribobio, China). $\beta$-actin and U6 were used as the endogenous control for the relative expression of mRNA and miRNA respectively. The specific primers used are listed in Table S1. The relative expression was calculated by the $2^{-\Delta \Delta C T}$ method.

\section{Actinomycin D and RNase $R$ treatment}

Transcription was prevented by the addition of 2 $\mathrm{mg} / \mathrm{mL}$ actinomycin D or DMSO (Sigma-Aldrich, USA) as the negative control to the cell culture medium. After treatment in the indicated time, the RNA expression levels of circMETTL3 and METTL3 were detected by qRT-PCR. For RNase R treatment, the original RNAs extracted from ZR-75-1 or SUM1315 cells were divided into two equal parts, one for RNase R treatment (RNase R) and the other for non-treatment (mock). A total of $2 \mathrm{mg}$ RNA was incubated for $30 \mathrm{~min}$ at $37^{\circ} \mathrm{C}$ with or without $3 \mathrm{U} / \mathrm{mg}$ RNase R (Epicenter Technologies, USA). The internal reference ( $\beta$-actin) in the mock group was used as the calculation standard.

\section{Cell transfection}

Transfections were carried out using the Lipofectamine 3000 Reagent (Invitrogen, USA) following the manufacturer's protocol. The small interfering RNAs (siRNAs) targeting circRNA were designed and synthesized by Ribobio (Guangzhou, China). miRNA mimics, miRNA inhibitors and their negative controls were designed and synthesized by GenePharma (Shanghai, China). Commercially available lentiviral vectors containing circMETTL3 sequence was constructed by Hanheng (Shanghai, 
China). Stable cells were selected using puromycin 5 $\mu \mathrm{g} / \mathrm{mL}$. The sequences of siRNAs and RNA oligonucleotides were listed in Table S2.

\section{Cell proliferation assay}

Cell proliferation assays were conducted by using Cell Counting Kit-8 (CCK-8) (Vazyme, China) in accordance with the manufacturer's instructions. 2000 cells were seeded into each well of 96-well plate with $100 \mu \mathrm{L}$ DMEM supplemented with $10 \%$ FBS. At the indicated time point of everyday, the medium was exchanged by $100 \mu \mathrm{L}$ DMEM with CCK-8 $(90 \mu \mathrm{L}$ DMEM and $10 \mu \mathrm{L}$ CCK-8) and the cells were incubated for 2h. Absorbance was detected spectrophotometrically at $450 \mathrm{~nm}$.

\section{Colony formation assay}

A total of 500 cells were plated in a six-well plate and maintained in DMEM medium containing 10\% FBS for approximately 2 weeks. Proliferating colonies were fixed with methanol and stained with $1 \%$ crystal violet (Beyotime, China). The number of colonies was counted by observing the proliferation of single cell.

\section{5-ethynyl-2'-deoxyuridine (EdU) incorporation assay}

Cell proliferation was measured via the EdU assay (Beyotime, China). The cells were cultured in 96-well plates $\left(2 \times 10^{4}\right.$ cells/well) with DMEM $(10 \%$ FBS) for $24 \mathrm{~h}$, incubated with $50 \mu \mathrm{M}$ EdU at $37^{\circ} \mathrm{C}$ for 2 $\mathrm{h}$, treated with $4 \%$ paraformaldehyde and $0.5 \%$ Triton X-100 and stained with $1 \times$ Apollo ${ }^{\circledR}$ reaction cocktail for $30 \mathrm{~min}$. Lastly, nuclei were stained with DAPI, and the cells were visualized under a fluorescence microscope.

\section{Cell wound-healing assay}

Cell wound-healing assay was used to evaluate cell migration. Cells were seeded in 6-well plates and cultured to the subfusion state. Then linear scratch wounds were created by $200 \mu \mathrm{L}$ sterile pipette tip. After washing three times with PBS, images were captured by inversion fluorescence microscopy at 0 and $48 \mathrm{~h}$ (Olympus, Japan).

\section{Transwell assay}

Transwell chambers with a membrane pore size of $8 \mathrm{~mm}$ were coated without or with Matrigel (BD Biosciences, USA). $200 \mu \mathrm{L}$ cell suspension in serum-free DMEM was added to the upper chambers, whereas $600 \mu \mathrm{L}$ medium containing 10\% FBS was used in the lower chamber. After incubation for $24 \mathrm{~h}$ or $48 \mathrm{~h}$, the cells were stained with crystal violet (Beyotime, China) to assess migration or invasion respectively.

\section{Fluorescence in situ hybridization (FISH)}

FISH assay was executed to observe the location of circMETTL3 in breast cancer cells. Briefly, probes for circMETTL3 were synthesized with 3'-Cy3-modification (RiboBio, China), and the cells were immobilized with $4 \%$ paraformaldehyde, followed by $0.5 \%$ Triton X-100. Then cells were incubated with $200 \mu \mathrm{L}$ of hybridization which contain corresponding probe overnight at $37^{\circ} \mathrm{C}$. After that, the cellular DNA was stained with DAPI and photographed by confocal microscope.

\section{Dual-luciferase reporter assay}

The putative miR-31-5p binding sequences in circMETTL3 or CDK1-3'-untranslated Regions(UTR) and their mutant of the binding sites were synthesized and cloned to downstream of the luciferase gene in the pmirGLO luciferase vector (Promega, USA). We then co-transfected the pmirGLO reporter gene with miR-31-5p mimics or miR-31-5p NC. The relative luciferase activity was measured with the DualLuciferase reporter assay system (Promega, USA).

\section{RNA immunoprecipitation (RIP)}

An RNA immunoprecipitation (RIP) assay was conducted in breast cancer cells using the Magna RIP RNA-Binding Protein Immunoprecipitation Kit (Millipore, Billerica, MA, USA) according to manufacturer's instructions. Anti-Argonaute2 (AGO2) antibody (Abcam, USA, ab186733) or NC rabbit IgG (Cell Signaling Technology, USA, 7076P2) were used. Briefly, cells were collected and lysed by the RIP lysis buffer. Then the corresponding antibodies were added into the cleared lysates and incubated in the magnetic beads' suspension with rotating overnight at $4{ }^{\circ} \mathrm{C}$. Precipitate was digested with proteinase $\mathrm{K}$ buffer, and then qRT-PCR was used to measure the levels of circMETTL3 transcripts in the AGO2 or IgG immunocomplexes.

The m6A RIP was performed as previously described with some modifications. Briefly, $3 \mu \mathrm{g}$ of anti-m6A antibody (Abcam, USA, ab208577) was conjugated to protein $A / G$ magnetic beads overnight at $4{ }^{\circ} \mathrm{C}$. And then the antibody conjugated beads were incubated with the antibody in IP buffer with RNase inhibitor and protease inhibitor. The interacting RNAs were isolated and detected by qRT-PCR.

\section{RNA pull down assay}

A total of $1 \times 10^{7}$ breast cancer cells were harvested, lysed, and sonicated. The cell lysates were incubated with streptavidin-coated magnetic beads to pull down the biotin-labeled RNA complex at $25^{\circ} \mathrm{C}$ for $2 \mathrm{~h}$ to generate probe-coated beads. Cell lysate with circMETTL3 probe or oligo probe was incubated 
at $4{ }^{\circ} \mathrm{C}$ for one night. After washing with wash buffer, the RNA mix bound to the beads was eluted and the complex was purified with Trizol (Takara, China).

\section{Western blot analysis}

The total protein of breast cancer cells was exacted with RIPA buffer and separated by $10 \%$ SDSPAGE, then electransferred onto a PVDF membrane (Bio-Rad, USA). The membranes were blocked with $5 \%$ skimmed milk powder and incubated with primary antibodies against METTL3 (1:1000, Abcam, USA, ab195352), CDK1 (1:1000, Proteintech, USA, 19532-1-AP), $\beta$-actin (Proteintech, China, 20536-1-AP) at $4{ }^{\circ} \mathrm{C}$ overnight and then incubated with secondary antibodies (1:5000) (Cell Signaling Technology, USA, $7074 \mathrm{P} 2$ ) at room temperature for $2 \mathrm{~h}$. Finally, the bands were examined by Immobilob ${ }^{\mathrm{TM}}$ Western Chemiluminescent HRP Substrate (Millipore, USA).

\section{Animal experiment}

All animal experiments were conducted according to the guidelines of Institutional Animal Care and Use Committee of the Nanjing Medical University. Twelve female BALB/c nude mice (aged 4 weeks, $18-22 \mathrm{~g}$ ) were randomly divided into 2 groups. Stable circMETTL3-expression SUM1315 cells or control cells $\left(1 \times 10^{6}\right.$ cells in $0.1 \mathrm{~mL}$ PBS $)$ was subcutaneously injected into mammary fat pads of the mice and the growth of tumors was followed up every week. Tumor volume was measured every week using a caliper, calculated as (length $\times$ width $\left.^{2}\right) / 2$. After 4 weeks, mice were sacrificed and checked for final tumor weight.

\section{Statistical analysis}

The statistical analysis was performed by SPSS software (Version 25.0) and GraphPad Prism (Version 8.0), and presented as mean \pm standard deviation (SD). All data were analyzed by two-tailed Student's t-test. Each experiment was repeated at least three times, and $\mathrm{p}<0.05$ was considered statistically significant.

\section{Results}

\section{Characterization of circMETTL3 in breast cancer}

Firstly, we performed sanger sequencing to detect the junction site of circMETTL3 and found it formed the spliced mature sequence with a full length of 623 nt (Fig. 1A). We conducted PCR using cDNA and gDNA (genomic DNA) as templates with two sets of primers for circMETTL3. The convergent primers could amplify circMETTL3 using cDNA and gDNA as templates, while the divergent primers only amplified
circMETTL3 using cDNA as templates, and no amplifications were observed using gDNA templates (Fig. 1B). We next investigated the stability of circMETTL3. After treatment with actinomycin D or RNase R, total RNAs were harvested from ZR-75-1 and SUM1315 cells at the indicated time points. The qRT-PCR assay showed circMETTL3 was more stable than METTL3 (Fig. 1C-D). circMETTL3 was highly expressed in breast cancer cell lines (Fig. 1E). METTL3 was also highly expressed in the same set of cell lines (Fig. S1). Moreover, it was expressed higher in breast cancer tumor tissues than adjacent normal tissues significantly (Fig. 1F). These results indicated that circMETTL3 was consistently expressed in breast cancer and might play an important role in breast cancer progression.

\section{circMETTL3 promotes breast cancer cell proliferation in vitro and in vivo}

To explore the function of circMETTL3 in breast cancer, we knocked down circMETTL3 expression with small interfering RNAs (siRNA) and found circMETTL3 expression was inhibited, without affecting the parental gene METTL3 (Fig. 2A-B). CCK-8 assay showed that knockdown of circMETTL3 could inhibit breast cancer cell proliferation significantly (Fig. 2C), consistent with the results from colony formation assay (Fig. 2D) and EdU assay (Fig. 2E). Accordingly, overexpression of circMETTL3 could promote breast cancer cells proliferation ability (Fig. S2A-D). Moreover, overexpression of circMETTL3 could facilitate the tumorigenesis of breast cancer cells in vivo (Fig. 2F-H). circMETTL3 was overexpressed in the treated group compared with the control group without affecting METTL3 expression (Fig. S3A-B).

\section{circMETTL3 enhances breast cancer cells migration and invasion ability}

We next investigated the effect of circMETTL3 on breast cancer cells metastasis and invasion. The wound healing assay showed that downregulation of circMETTL3 could decrease breast cancer cells migration significantly (Fig. 3A-B); by contrast, overexpression of circMETTL3 could enhance breast cancer cells migration (Fig. S4A-C). In the Transwell assay, the number of migration cells decreased dramatically after the circMETTL3 expression was reduced (Fig. 3C). An opposite effect was detected in the breast cells when circMETTL3 was overexpressed (Fig. S4D, F). Moreover, reduced circMETTL3 expression could inhibit breast cancer cells invasion ability (Fig. 3D), but increasing circMETTL3 expression could reverse the effect (Fig. S4E, G). 
A

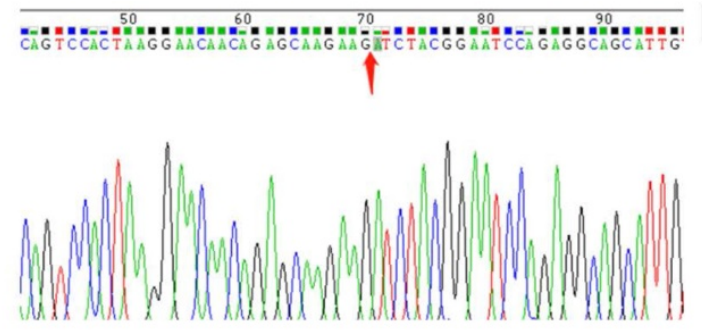

C

ZR-75-1

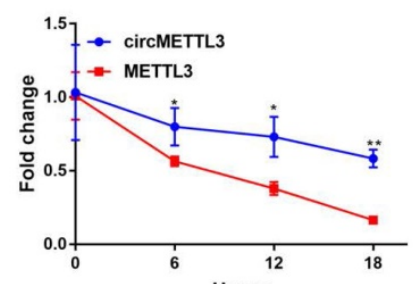

$\mathrm{E}$

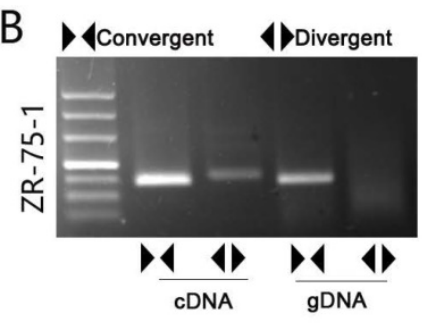

ZR-75-1
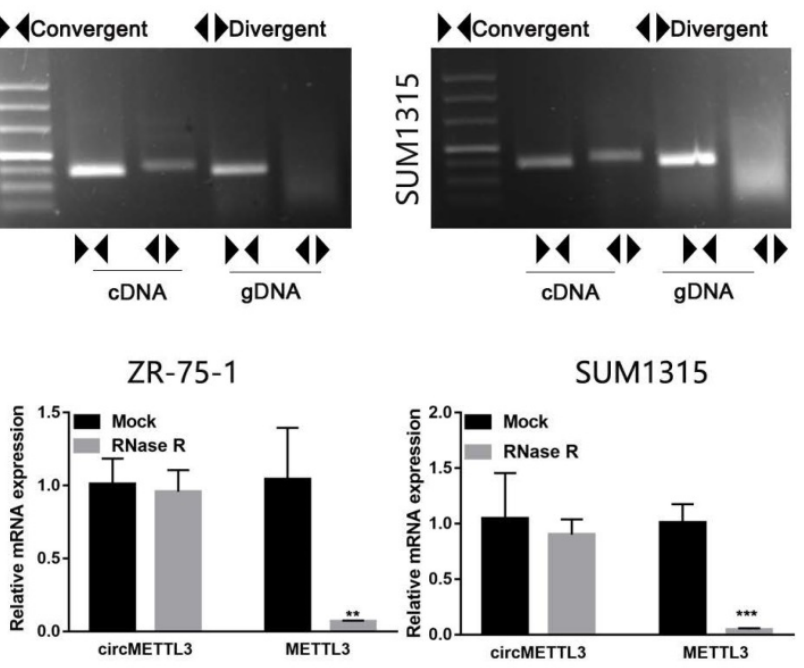

$\mathrm{D}$

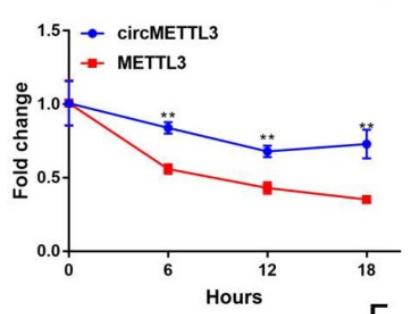

F
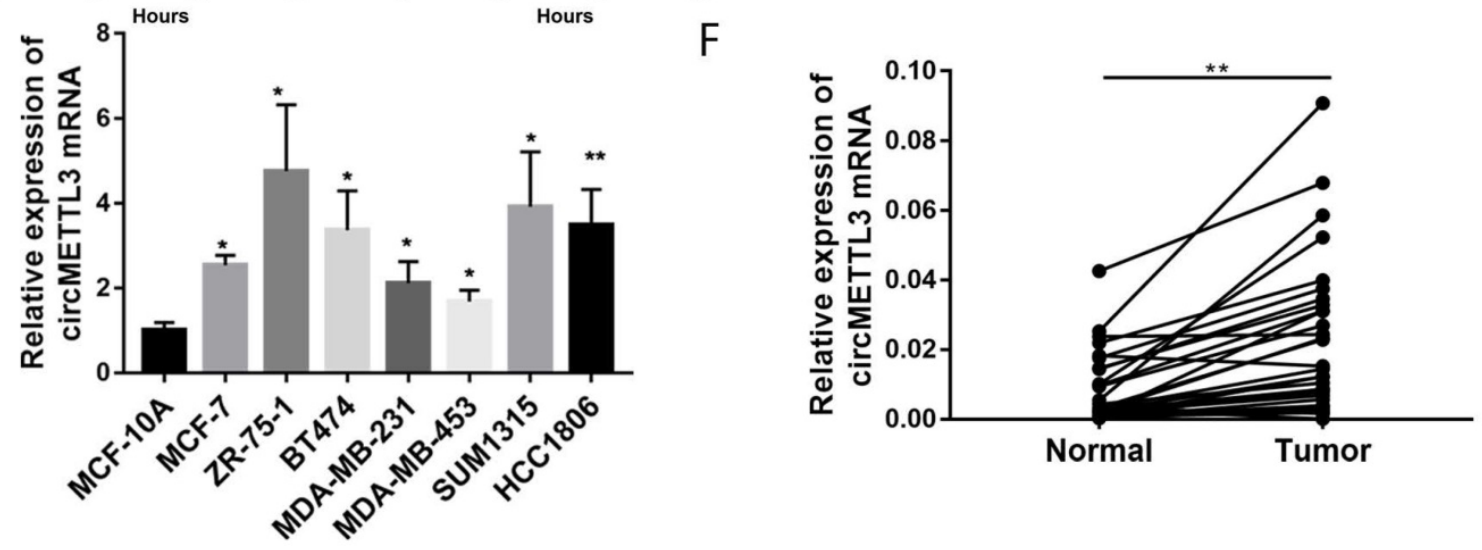

Figure 1. Characterization of circMETTL3 in breast cancer cells and tissues. A. Sanger sequencing-validated circular structure. Arrows indicated the junction site of circMETTL3. B. RT-PCR analysis for circMETTL3 and its linear transcript METTL3 in CDNA and gDNA in breast cancer cells. C. qRT-PCR analysis of circMETTL3 and METTL3 after treatment with actinomycin D at the indicated time points. D. qRT-PCR showed the expression of circMETTL3 and METTL3 mRNA in breast cancer cells treated with RNase R or mock control. E. Expression of circMETTL3 in the breast cancer cell lines and MCF-10A by qRT-PCR. F. Expression of circMETTL3 in breast cancer tissues and adjacent normal tissues. Data were shown as mean \pm SD. ${ }^{*} p<0.05, * * p<0.01, * * * p<0.001$.

\section{circMETTL3 acts as a sponge and targets miR-31-5p directly}

To dissect the underlying mechanism by which circMETTL3 promotes breast cancer proliferation and metastasis, we performed FISH assay to observe the subcellular localization of circMETTL3. The results showed circMETTL3 was mainly located in cytoplasm in breast cancer cells (Fig. 4A), suggesting circMETTL3 might act as miRNA sponge. Through bioinformatics analysis, we took the intersection of the miRNAs predicted in CircInteractome with context+ score percentile greater than 90, the circBank and starBase predictions (Fig. 4B), and combined the correlation between and the miRNAs expression and breast cancer patients prognosis. miR-31-5p and miR-769-5p might be sponged by circMETTL3. But knockdown of circMETTL3 had no effect on miR-769-5p expression. We identified miR-31-5p as the candidate sponged by circMETTL3. Survival curve showed that patients with high expression of miR-31-5p had better overall survival rate (Fig. 4C).
Moreover, knockdown of circMETTL3 could increase miR-31-5p expression in breast cancer cells (Fig. 4D) while overexpression of circMETTL3 decreased miR-31-5p expression in the previous animal tumors (Fig. S5A). Moreover, we detected correlation between miR-31-5p and circMETTL3 expression in breast cancer tissues and found the expression of miR-31-5 and circMETTL3 is negatively correlated (Fig. 4E). Then, dual-luciferase reporter assays were performed to measure the binding capacity between circMETTL 3 and miR-31-5p in breast cancer cells. The wild and mutant dual-luciferase reporter plasmids of circMETTL3 were constructed accordingly. The results indicated that miR-31-5p mimics reduced the luciferase activity of circMETTL3-WT luciferase reporter obviously, but not that of mutants (Fig. 4F). It is well known that miRNAs degrade mRNAs and inhibit translation in an AGO2-dependent manner via binding to their targets directly. We thus performed RIP assay and found that circMETTL3 was enriched in AGO2 immunoprecipitates in breast cancer cells (Fig. $4 \mathrm{G})$. Furthermore, we performed pull down assay and 
found that miR-31-5p was abundantly pulled down by circMETTL3 probe, compared with negative control probe in breast cancer cells (Fig. 4H). Overall, these results suggested that circMETTL3 could act as a sponge and targets miR-31-5p directly.

\section{miR-31-5p partly reverses the effects caused by circMETTL3 in breast cancer cells}

We then explored the effect of miR-31-5p on the role of circMETTL3 in breast cancer. The CCK-8 assay showed that inhibition of miR-31-5p expression partly reversed the decrease in breast cancer cells proliferation caused by knockdown of circMETTL3 (Fig. 5A-B). Number of colonies (Fig. 5C) and EdU positive cells also increased significantly (Fig. 5D-E). Moreover, downregulated miR-31-5 $p$ could promote migration area inhibited by knockdown of circMETTL3 (Fig. 6A-C). Number of migration and invasion cells increased with inhibition of miR-31-5p (Fig. 6D-G). Overall, these results confirmed circMETTL3 promoted breast cancer progression by acting as a sponge of miR-31-5p.
A

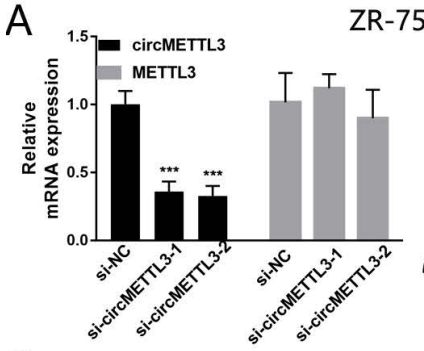

C

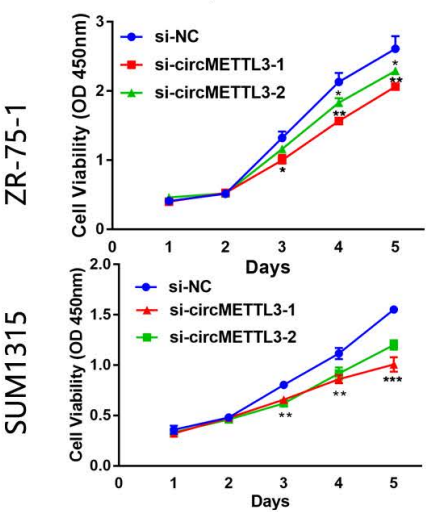

si-1 si-2

B

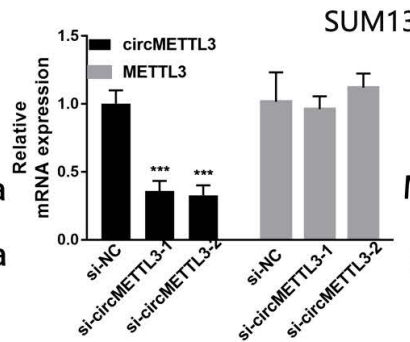

SUM1315

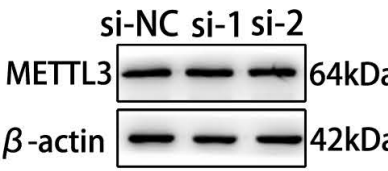

D
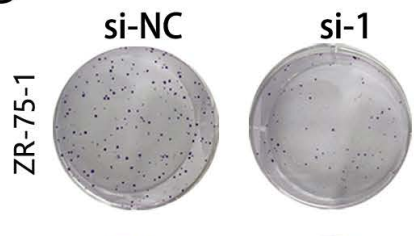

si-2
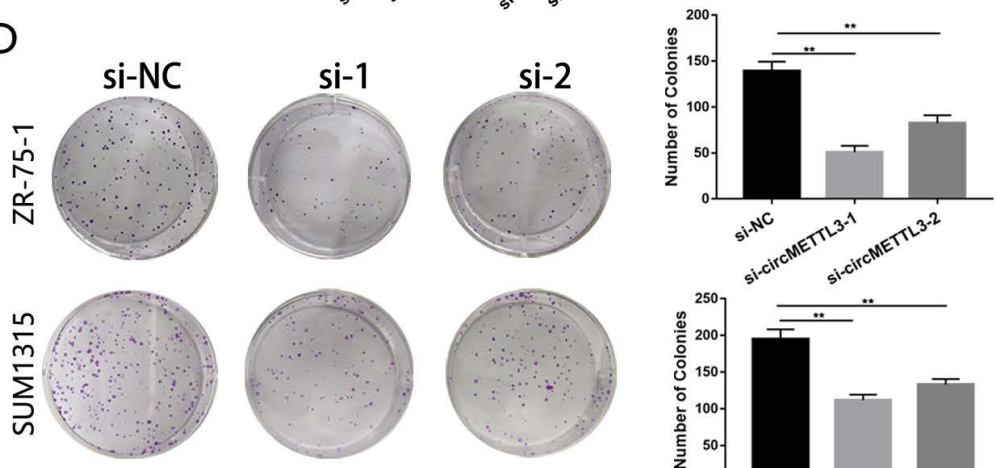

METTL3 $\approx=64 \mathrm{kDa}$

$\beta$-actin $-2 \mathrm{k} 2 \mathrm{kDa}$

$E$

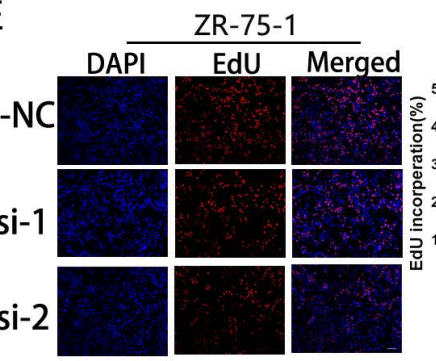

$\mathrm{F}$
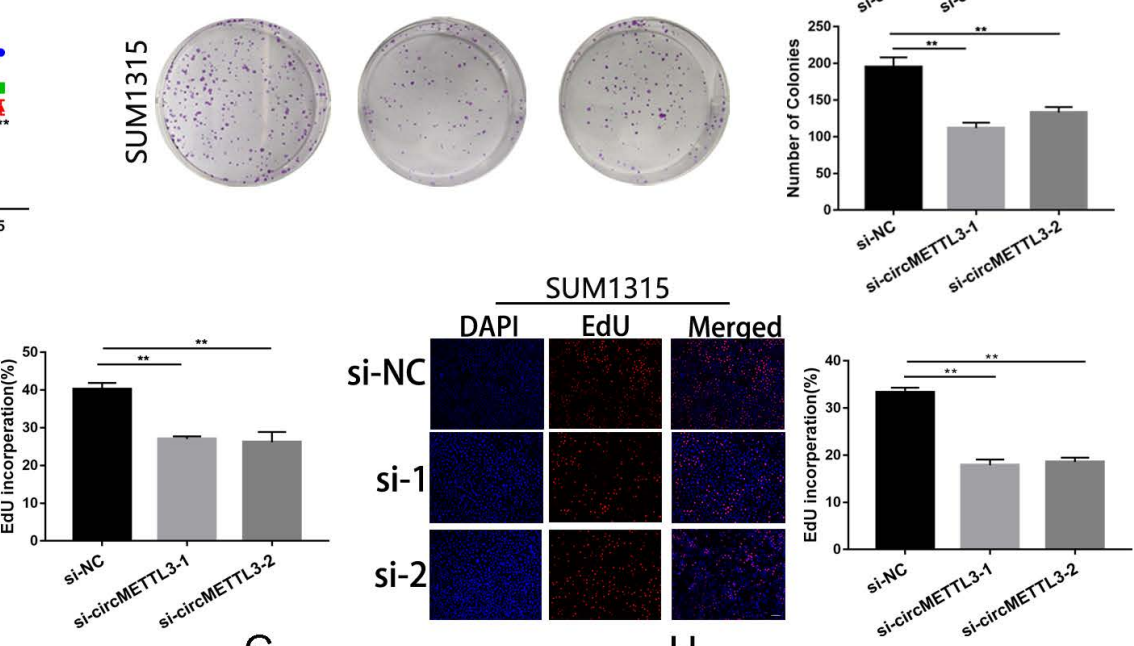

G
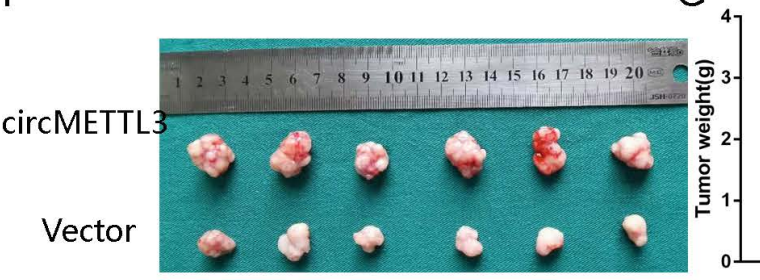

$\mathrm{H}$

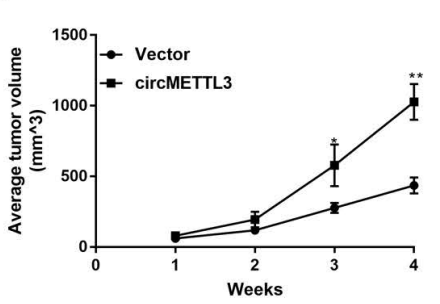

Figure 2. Knockdown of circMETTL3 inhibits breast cancer cell proliferation in vitro and in vivo. A-B. QRT-PCR and western blot analysis of the transfection efficacy of siRNA on circMETTL 3 and METTL 3 expression in ZR-75-1 (A) and SUM1315 (B) cell lines after 48 h transfection. C. The proliferation ability of ZR-75-1 and SUM1315 cells lines transfected with siRNAs was determined by CCK-8 assays. D. The colony formation results of ZR-75-1 and SUM1315 cells lines transfected with siRNAs. Colonies $>50$ mm were counted. E. EdU assays results of ZR-75-1 and SUM1315 cells lines transfected with siRNAs. Scale bar, $100 \mu \mathrm{m}$. Blue indicates DAPI, red indicates EdU, si-NC indicates negative control, si-1 indicates si-circMETTL3-1,si-2 indicates si-circMETTL3-2. F. Representative images of xenograft tumors of circMETTL3 overexpression (circMETTL3) and control (Vector) group $(n=6)$. G. Tumor weights in these two groups were shown. $H$. Tumor volumes were measured weekly in all mice that received subcutaneous injections. Data were shown as mean \pm SD. ${ }^{*} p<0.05$, ${ }^{*} p<<0.01$, ${ }^{*} * *_{p}<0.001$. 
A

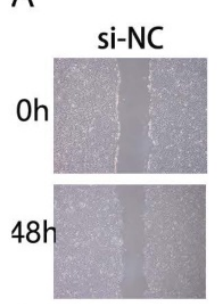

C
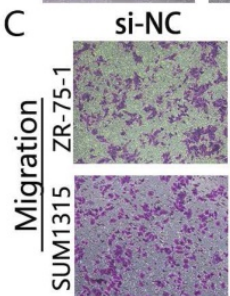

ZR-75-1

si-1

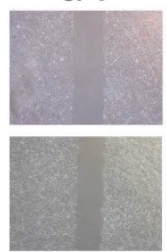

si-1

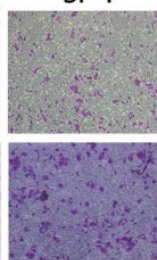

si-2

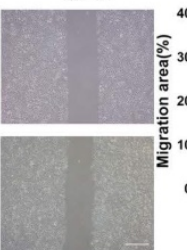

si-2

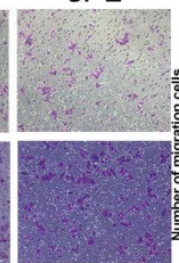

B

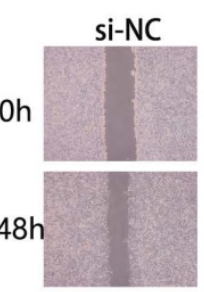

D
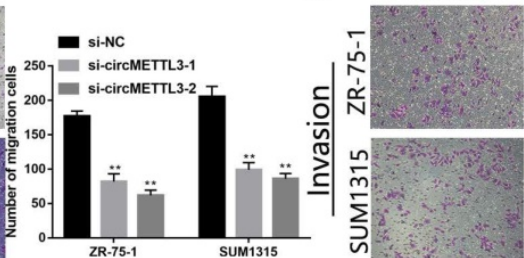

SUM1315

si-1
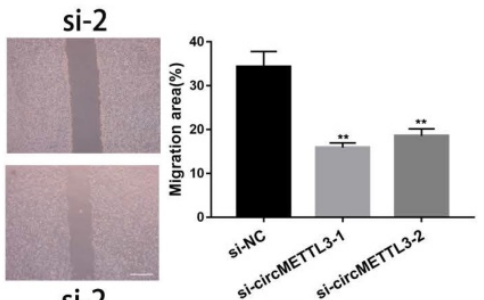

si-2 si-1

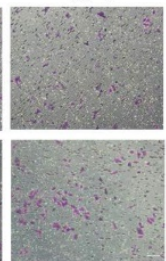

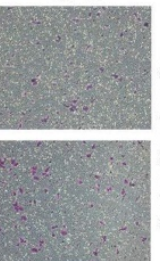

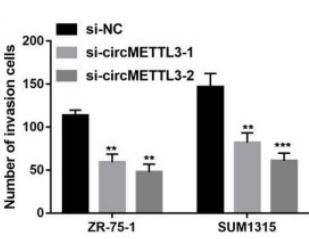

Figure 3. circMETTL3 enhances breast cancer cells migration and invasion ability. A-B. The wound healing assays were performed to assess the effect of circMETTL3 on cell motility at 0 and $48 \mathrm{~h}$ in ZR-75-1 (A) and SUM1315 (B) cell lines. Scale bar, $100 \mu \mathrm{m}$. C-D. The representative images of migrated (C) and invaded (D) ZR-75-1 and SUM1315 cell lines after circMETTL3 knockdown. Scale bar, $100 \mu \mathrm{m}$.si-NC indicates negative control, si-1 indicates si-circMETTL3-1,si-2 indicates si-circMETTL3-2. Data were shown as mean $\pm \mathrm{SD},{ }^{*} \mathrm{p}<0.05$, **p $<0.01$, *** $\mathrm{p}<0.001$

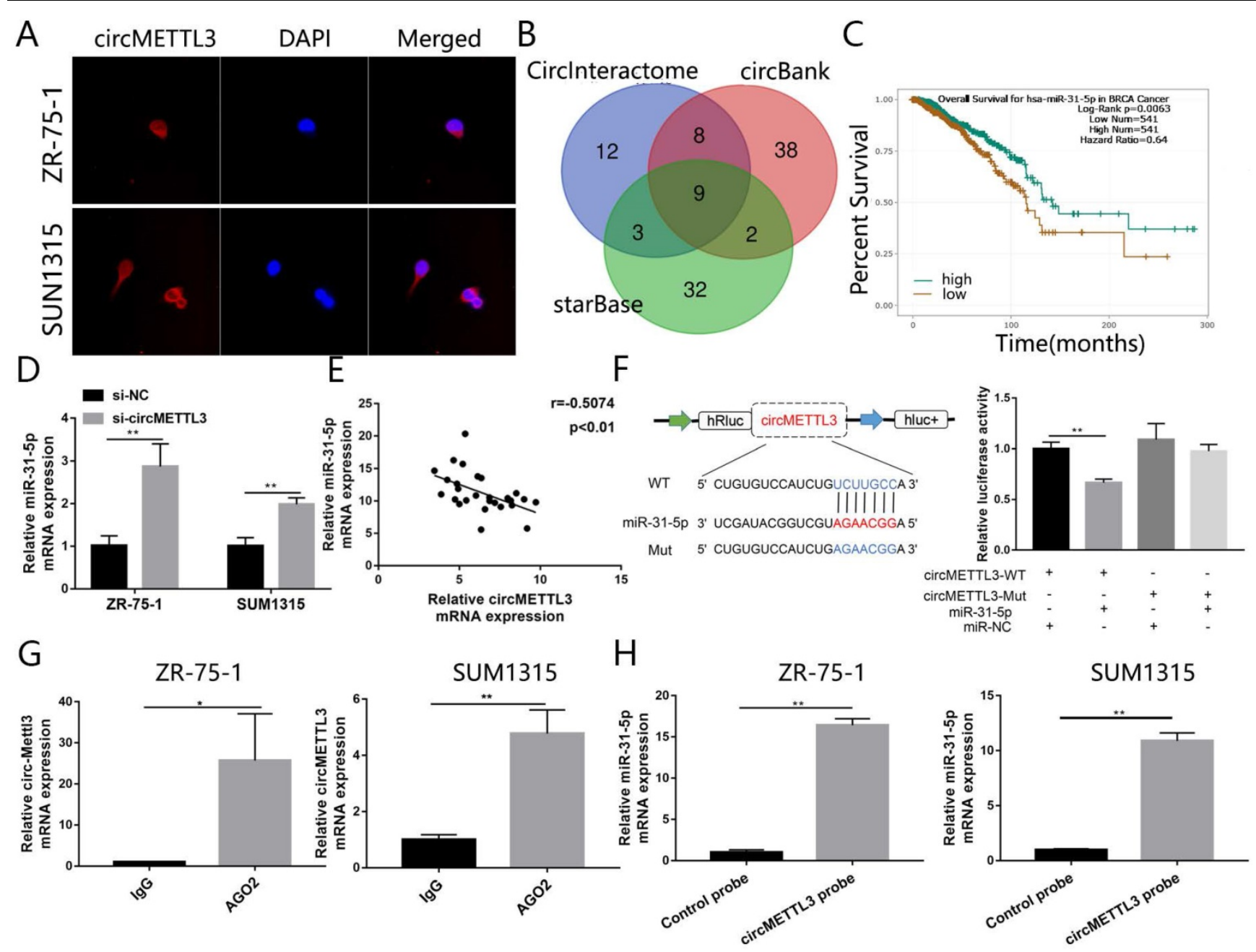

Figure 4. circMETTL3 acts as the sponge of miR-31-5p in breast cancer. A. FISH revealed the subcellular localization of circMETTL3. Red indicates circMETTL3, bule indicates DAPI. B. The Venn-diagram showed the potential miRNAs sponged by circMETTL3 predicted by combination of three bioinformatics algorithms (Circlnteractome, circBank, starBase). C. Kaplan-Meier survival analysis showed the correlation between the expression of miR-31-5p and the overall survival of breast cancer patients based on TCGA. D. Knockdown of circMETTL3 increased expression of miR-31-5p in breast cancer cell lines. E. Correlation between circMETTL3 and METTL3 expression in breast cancer tissues. F. Schematic representation of circMETTL3-WT and circMETTL3-Mut luciferase reporter vectors (left). Luciferase reporter assay indicated the binding capacity of circMETTL3 with miR-31-5p (right). G. Anti-AGO2 RIP experiments were performed to detect circMETTL3 expression in breast cancer cell lines. ZR-75-1 and SUM1315 cells lysates were immunoprecipitated with AGO2 or IgG antibody and analyzed by using QRT-PCR to detect circMETTL3 transcript levels. H. RNA pull-down was performed to detect the enrichment of miR-31-5p in breast cancer cell lines. Data were shown as mean \pm SD, $*<0.05, * * p<0.01, * * * p<0.001$. 
A

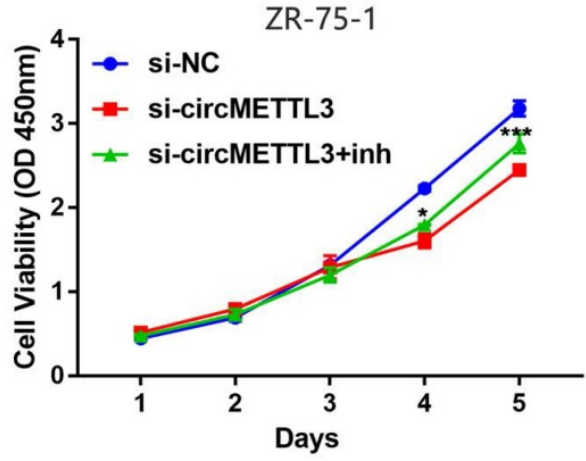

B

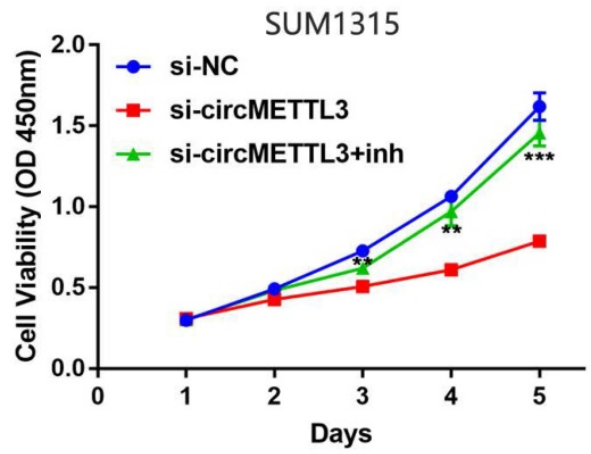

C

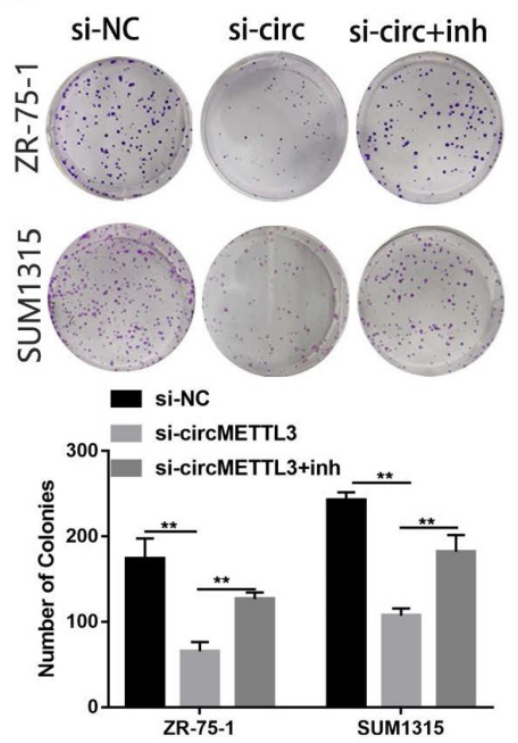

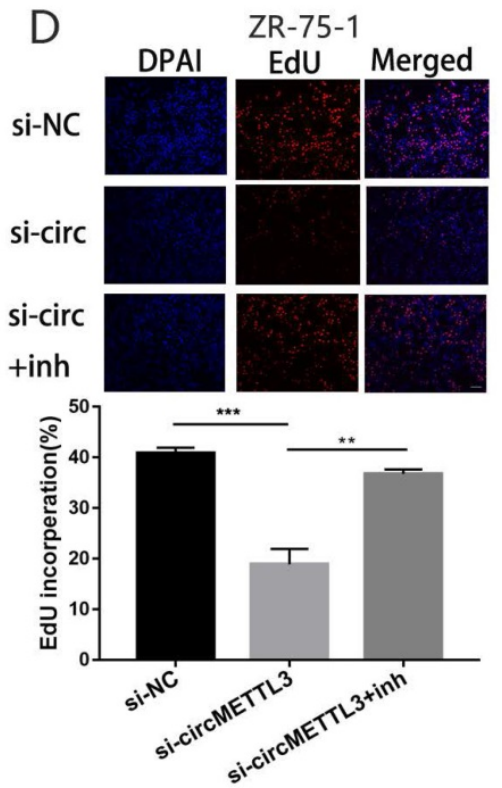

E
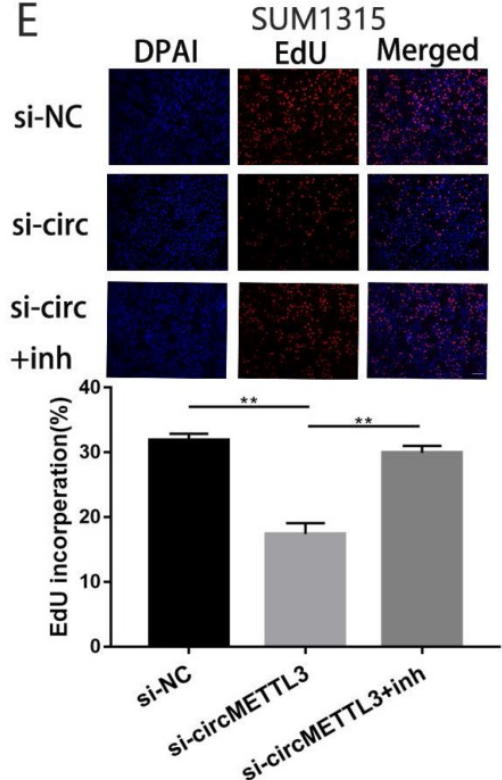

Figure 5. miR-31-5p partly reverses the cell proliferation caused by circMETTL3 in breast cancer cells lines. A-B. Cell proliferation of ZR-75-1 (A) and SUM1315 (B) cell lines transfected with negative control (si-NC), si-circMETTL3 (si-circ) or circMETTL3+miR-31-5p inhibitor (si-circMETTL3+inh) was determined by CCK-8. C: Cell proliferation of ZR-75-1 and SUM1315 cell lines transfected with negative control, si-circMETTL3 or circMETTL3+miR-31-5p inhibitor was determined by colony formation assay. Colonies > $50 \mathrm{~mm}$ were counted. D-E. Cell proliferation of ZR-75-1 (D) and SUM1315 (E) cell lines transfected with negative control, si-circMETTL3 or circMETTL3+miR-31-5p inhibitor. Blue indicates DAPI, red indicates EdU.Scale bar, $100 \mu \mathrm{m}$. Data were shown as mean \pm SD, ${ }^{*} \mathrm{p}<0.05$, $* * \mathrm{p}<0.01$, ${ }^{* * *} \mathrm{p}<0.001$.

\section{CDK1 is a direct target of miR-31-5p in breast cancer}

We used three algorithms (mirDIP, Targetscan and miTarBase) and GEPIA to identity the putative targeted genes of miR-31-5p. The overlap of the targeted genes predicted by the three algorithms and the 300 genes upregulated in breast cancer in GEPIA showed that CDK1 might be the targeted gene of miR-31-5p (Fig. 7A). Forced miR-31-5p expression decreased both CDK1 mRNA and protein expression, while inhibition of miR-31-5p reversed the effect (Fig. 7B-C).We then performed dual-luciferase reporter assays and found upregulated miR-31-5p significantly inhibited luciferase activity of CDK1-WT rather than that of CDK1-Mut (Fig. 7D). Moreover, knockdown of circMETTL3 inhibited CKD1 expression in breast cancer cells (Fig. 7E) while overexpression of circMETTL3 increased CKD1 expression (Fig. S5B-C), but inhibition of miR-31-5p partly reversed the effect (Fig. 7F-G). These results proved that circMETTL3 increased CDK1 expression by sponging miR-31-5p to promote breast cancer progression.

\section{circMETTL3 is upregulated in a m6A- dependent manner}

Through the circBank, we found that cicMETTL3 might have m6A modification (Fig. 8A). We detected correlation between METTL3 and circMETTL3 expression in breast cancer tissues and found the expression of circMETTL3 and METTL3 is positively correlated (Fig. 8B). MeRIP assay showed circMETTL3 was highly recruited in $\mathrm{m} 6 \mathrm{~A}$ precipitated fraction (Fig. 8C). Previous studies showed m6As control circZNF609 biogenesis [27]. We then explored whether the m6A modification of circMETTL3 affected its expression. The results showed knockdown of METTL3 decreased circMETTL3 expression (Fig. 8D). Moreover, METTL3 increased CDK1 expression in the absence of circMETTL3 (Fig. S6A-B), and the result was consistent with our previous study [21]. As circMETTL3 was derived from METTL3, we knock 
downed METTL14 or FTO expression to regulate m6A levels in circMETTL3 to avoid changes in circMETTL3 expression caused by the off-target effects of knockdown of METTL3. Downregulated METTL14 decreased circMETTL3 expression and increased pre-METTL3 expression, while decreased FTO expression caused opposite effect (Fig. 8E-F). Moreover, m6A modification of circMETTL3 increases its expression (Fig. 8G).

\section{Discussion}

In the present study, we characterized a novel circRNA, named as circMETTL3. The circRNA is a 623 nt transcript originated from the exon- 2 and exon- 3 of METTL3 gene. It was highly expressed in breast cancer cell lines and breast cancer tumor tissues. Moreover, circMETTL3 could promote proliferation and metastasis abilities of breast cancer cells, while knockdown of circMETTL3 showed an opposite effect. Though the cell lines ZR-75-1 and SUM1315 had different estrogen receptor status, circMETTL3 performed oncogenic function in both of the two cell lines, indicating the oncogenic role of circMETTL3 are estrogen receptor status-independent. We then found circMETTL3 mainly located in cytoplasm breast cancer cells and contained miRNA response element of miR-31-5p through bioinformatics analyses. Further, dual-luciferase reporter, anti-AGO2 RIP and RNA pull-down assays confirmed that circMETTL3 could interact with miR-31-5p directly and knockdown of circMETTL3 increased miR-31-5p expression. We also found that miR-31-5p was positively correlated with patients' overall survival based on the TCGA dataset. Through bioinformatics analysis, we identified CDK1 as a potential target of miR-31-5p, which was confirmed by luciferase reporter assay. CDK1, a member of the CDKs, plays a critical role in different types of cancers [21, 28-30]. The other study showed CDK1 acted as a novel prognostic indicator for early breast cancer [31]. According to our data, downregulated of circMETTL3 decreased CDK1 expression. These findings suggest

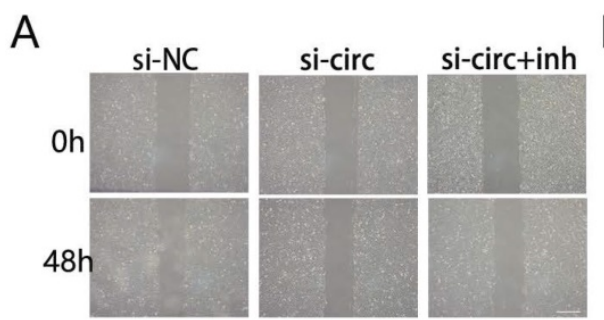

ZR-75-1

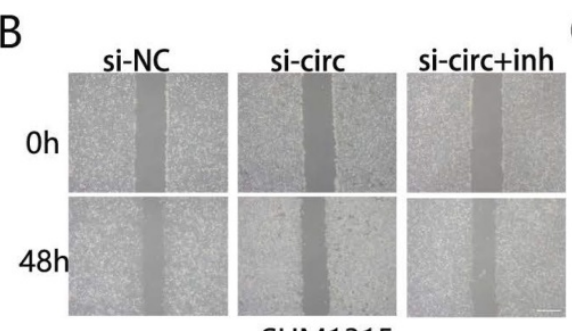

SUM1315

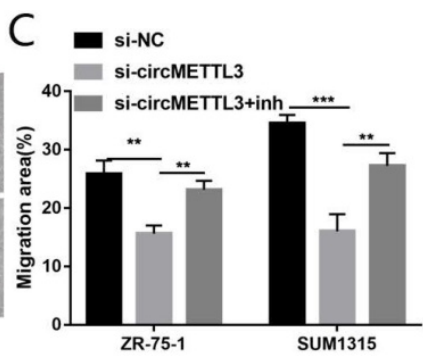

E
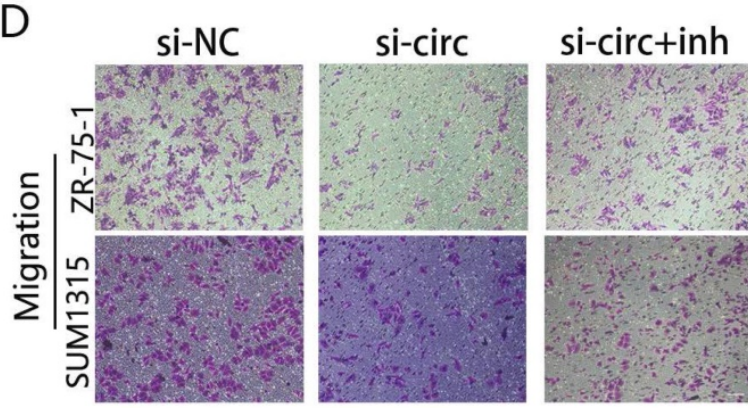

$\mathrm{F}$
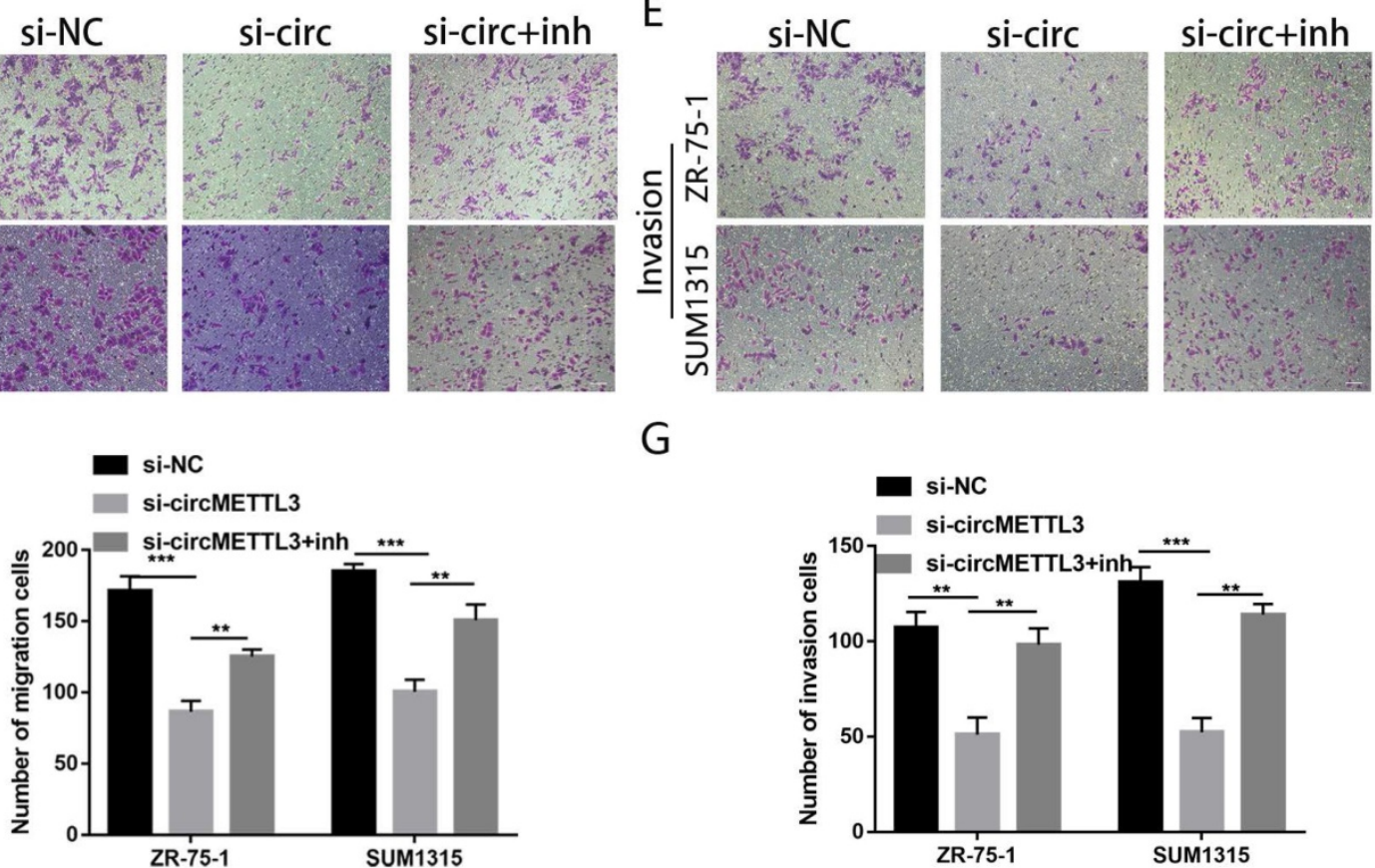

G

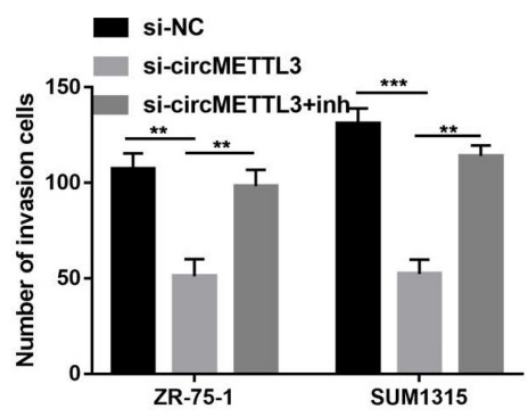

Figure 6. miR-31-5p partly reverses the metastasis caused by circMETTL3 in breast cancer cells lines. A-C. The wound healing assays were used to detect the ZR-75-1(A) and SUM1315 (B) cell lines transfected with negative control (si-NC), si-circMETTL3 (si-circ) or circMETTL3+miR-31-5p inhibitor (si-circ+inh) migration ability. Scale bar, $100 \mu \mathrm{m} \mathrm{D-G}$. Transwell assay was performed to determine the migration (D and F) and invasion (E and G) abilities of ZR-75-1 and SUM1315 cell lines transfected with negative control, si-circMETTL3 or circMETTL3+miR-31-5p inhibitor. Scale bar, $100 \mu \mathrm{m}$. Data were shown as mean \pm SD, *p<0.01, **p $<0.01$, *** $\mathrm{p}<0.001$. 
circMETTL3 acts as an oncogene in breast cancer progression and become a potential diagnostic and therapy target for breast cancer patients. Previous studies showed circRNAs might perform biological functions in tumors through different mechanisms, including act as a sponge of miRNAs [32], interact with RNA binding proteins, enhance targeted gene mRNA stability, influence host gene, or encode peptides [10,33]. For example, circANKS1B promoted breast cancer metastasis via acting as a sponge of miRNAs [12]. circBMPR2 inhibited breast cancer progression via circBMPR2/miR-553/USP4 axis [34]. circNSUN2 interacted with YTHDC1 to promote its cytoplasmic export and stabilized HMGA2 mRNA via interacting with IGF2BP2 [35]. circKIAA1429 accelerated hepatocellular carcinoma advancement through enhancing ZEB1 mRNA stability [26].
circSMARCA5 terminated the transcription of SMARCA5 to inhibit DNA damage repair [14]. circSHPRH suppressed glioma tumorigenesis by encoding a novel protein [36]. circFNDC3B encoded a novel protein to inhibit colon cancer progression [37]. In our study, results illustrated circMETTL3 acts as a sponge of miR-31-5p to increase CKD1 expression, in line with previous studies $[11,12,38]$.

Previous studies showed circRNAs might act as a sponge to increase its host gene expression [39-41] or decrease the parental gene expression by terminating the transcription of the parental gene [14]. However, the relation of circMETTL3 and its parental gene, METTL3, was still elusive. Several studies showed METTL3 promote different cancers through affecting its target genes and non-coding RNAs in an m6A dependent manner [24, 25, 42, 43]. We then explored
A

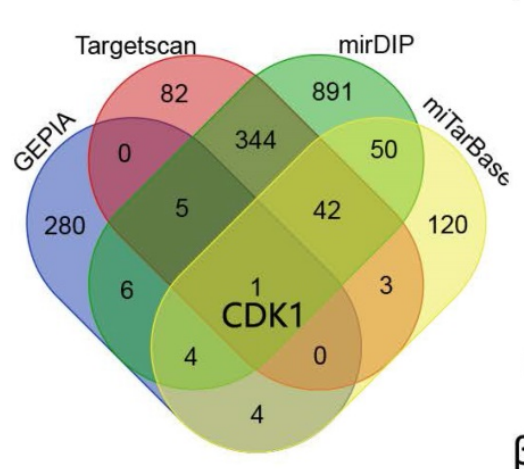

B
C

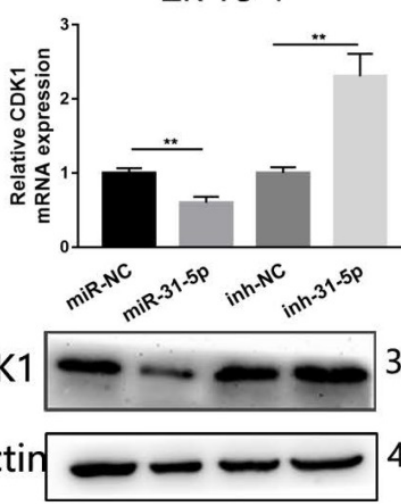

$\mathrm{F}$
D

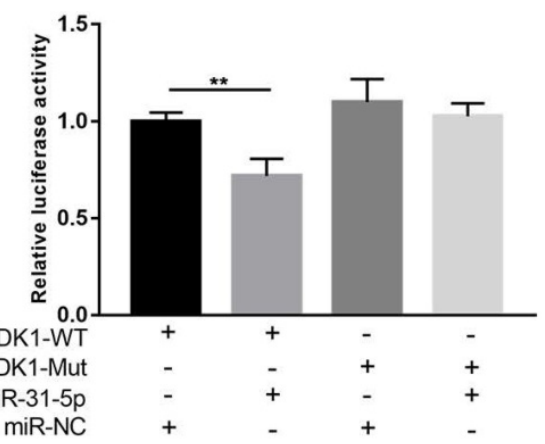

$\mathrm{E}$

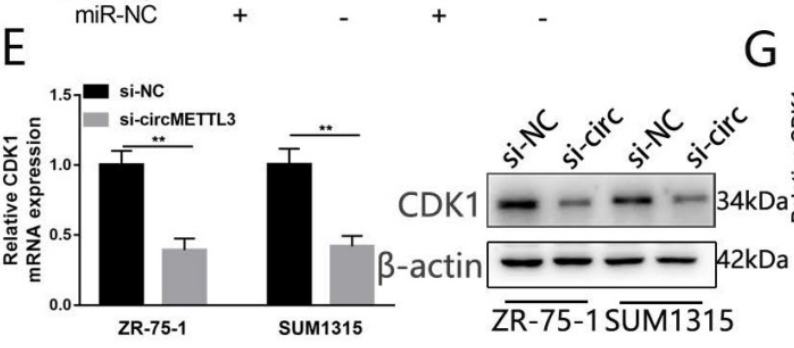

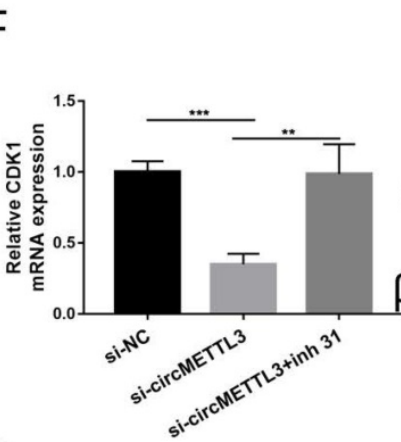

42kDa $\beta$-actin
SUM1315

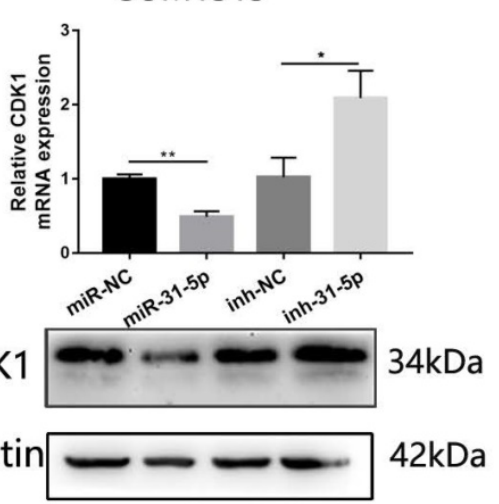

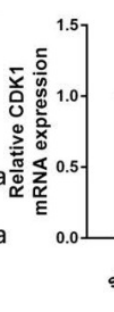

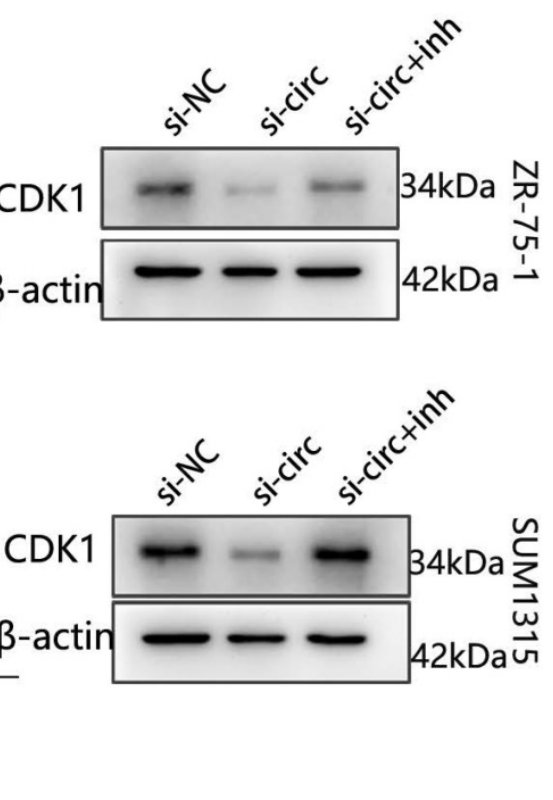

Figure 7. CDK1 is the target of miR-31-5p and indirectly regulated by circMETTL3. A. The Venn-diagram indicates the number of genes that overlapped in three publicly available bioinformatics algorithms (Targetscan, mirDIP, miTarbase) and top 300 upregulated genes in breast cancer downloaded from GEPIA (GEPIA). B-C: The mRNA and protein expression levels of CDK1 in ZR-75-1 (B) and SUM1315(C) cell lines transfected with miR-31-5p mimic (miR-31-5p) or miR-31-5p inhibitor (inh-31-5p). D. The relative luciferase activities were detected in 293T cells after transfected with CDK1 3'UTR-WT or CDK1 3'UTR-Mut and miR-31-5p mimics or miR-NC, respectively. E. Expression of CDK1 in breast cancer cells after knockdown of circMETTL3. F-G: qRT-PCR and western blot were excused to detect CDK1 expression in ZR-75-1 (F) and SUM1315 (G) cell lines transfected with negative control (si-NC), si-circMETTL3 (si-circ) or circMETTL3+miR-31-5p inhibitor(si-circ+inh). Data were shown as mean \pm SD, ${ }^{*} \mathrm{p}<0.01,{ }^{* *} \mathrm{p}<0.01,{ }^{* * *} \mathrm{p}<0.001$. 
whether METTL3 affected circMETTL3 expression by the mechanism of m6A modification. As circMETTL3 was derived from METTL3, we knockdown METTL14, which helps METTL3 to recognize its RNA substrates, or FTO, function as m6A demethylases, to regulate m6A levels in circMETTL3 to avoid changes in circMETTL3 expression caused by the off-target effects of knockdown of METTL3. We found that downregulated METTL14 could decrease circMETTL3 expression and increase pre-METTL3 expression, while decreased FTO expression caused opposite effect. These results indicated m6A modification might affect circMETTL3 expression, in line with previous study METTL3 affect circ-ZNF609 biogenesis in an m6A dependent manner [27]. We also found that knockdown of circMETTL3 expression with small interfering RNA could not affect the parental gene METTL3 expression. Moreover, the expression of METTL3 and circMETTL3 are positively correlated in breast cancer tissues. All the results suggested that METTL3 might increase circMETTL3 expression in an m6A dependent manner. It illustrated METTL3 increased circMeETTL3 expression, derived from its own, to promote breast cancer progression by its methyltransferase activity [24, 25]. Another component in m6A methyltransferase enzymes, KIAA1429 and its circKIAA1429 were also highly expressed and promoted hepatocellular carcinoma advancement [26, 44], showing the similar mode as METTL3 and circMETTL3 in breast cancer. Moreover, parental gene could regulate corresponding circRNAs expression through their own function, besides acting as sponges of miRNAs or terminating the transcription of the parental gene, providing a new insight into the relationship between circRNAs and their parental gene.

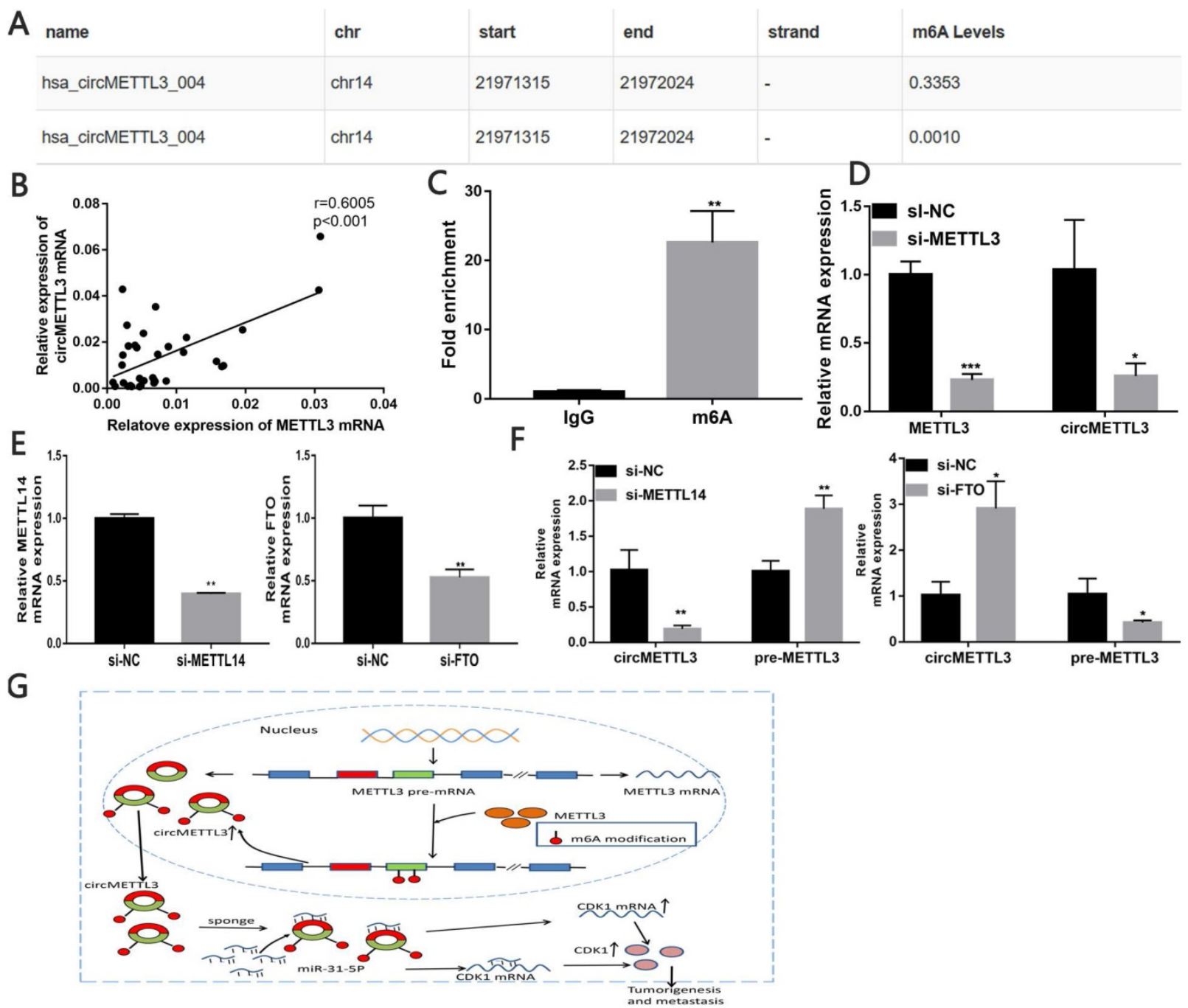

Figure 8. circMETTL3 is upregulated in a m6A-dependent manner. A. Possibility of m6A modification of circMETTL3 B. Correlation between circMETTL3 and METTL3 expression in breast cancer tissues. C. Anti-m6A RIP experiments were performed to detect circMETTL3 expression. D. qRT-PCR showed METTL3 and circMETTL3 expression after knockdown of METTL3. E. qRT-PCR analysis of the transfection efficacy of siRNA on Mettl14 and FTO. F. qRT-PCR showed circMETTL3 and pre-METTL3 expression after knockdown of METTL14 or FTO. G. Schematic diagram illustrates the mechanism of circMETTL3 up-regulated in a m6A-dependent manner to promote breast cancer progression through circMETTL3/miR-31-5p/CDK1 axis. Data were shown as mean \pm SD, $*_{p}<0.05, * * p<0.01, * * *_{p}<0.001$. 


\section{Conclusion}

In conclusion, our results illustrated the vital role of circMETTL3 in the breast cancer progression. Mechanistically, circMETTL3 harbors miR-31-5p to upregulate CDK1 expression. Moreover, modification of circMETTL3 increases its expression. METTL3, the circMETTL3 host gene, is the most important part of methyltransferase complex and may increase circMETTL3 expression in an m6A-depenent manner. Thus, circMETTL3 might act as a potential therapeutic target for breast cancer.

\section{Abbreviations}

CircRNA: Circular RNA; m6A: N6-methyladenosine; qRT-PCR: Real-time quantitative polymerase chain reaction; cDNA: Complementary DNA; gDNA: Genomic DNA; 3'UTR: 3' -untranslated region; ceRNA: Competing endogenous RNA; FISH: Fluorescence in situ hybridization; miRNA: MicroRNA; RIP: RNA immunoprecipitation; RNAi: RNA interfere.

\section{Supplementary Material}

Supplementary figures and tables.

http://www.ijbs.com/v17p1178s1.pdf

\section{Acknowledgements}

This work was supported by the National Natural Science Foundation of China (81972486, 81802748, 81802644), the Key Medical Talents of Jiangsu Province (ZDRCA2016029) and '333' High-level Talents Training Project of Jiangsu Province (BRA2016505). Besides, the work was also funded by the International Cooperation Project of Jiangsu Provincial Science and Technology Department (BZ2018054) and the Priority Academic Program Development of Jiangsu Higher Education Institutions (PAPD) (JX10231801).

\section{Competing Interests}

The authors have declared that no competing interest exists.

\section{References}

1. Siegel RL, Miller KD, Jemal A. Cancer statistics, 2020. CA Cancer J Clin. 2020; 70: 7-30

2. DeSantis CE, Ma J, Gaudet MM, Newman LA, Miller KD, Goding Sauer A, et al. Breast cancer statistics, 2019. CA Cancer J Clin. 2019; 69: 438-51.

3. Ruan $\mathrm{H}$, Xiang $\mathrm{Y}$, Ko J, Li S, Jing $\mathrm{Y}$, Zhu X, et al. Comprehensive characterization of circular RNAs in $\sim 1000$ human cancer cell lines. Genome Med. 2019; 11: 55.

4. Kristensen LS, Andersen MS, Stagsted LVW, Ebbesen KK, Hansen TB, Kjems J. The biogenesis, biology and characterization of circular RNAs. Nat Rev Genet. 2019; 20: 675-91.

5. Liu L, Wang J, Khanabdali R, Kalionis B, Tai X, Xia S. Circular RNAs: Isolation, characterization and their potential role in diseases. RNA Biol. 2017; 14: 1715-21.
6. Zheng $X$, Huang $M$, Xing $L$, Yang $R$, Wang $X$, Jiang $R$, et al. The circRNA circSEPT9 mediated by E2F1 and EIF4A3 facilitates the carcinogenesis and development of triple-negative breast cancer. Mol Cancer. 2020; 19: 73.

7. Wang R, Zhang S, Chen X, Li N, Li J, Jia R, et al. EIF4A3-induced circular RNA MMP9 (circMMP9) acts as a sponge of miR-124 and promotes glioblastoma multiforme cell tumorigenesis. Mol Cancer. 2018; 17: 166.

8. $\mathrm{Ng} \mathrm{WL}$, Mohd Mohidin TB, Shukla K. Functional role of circular RNAs in cancer development and progression. RNA Biol. 2018; 15: 995-1005.

9. Arnaiz E, Sole C, Manterola L, Iparraguirre L, Otaegui D, Lawrie CH. CircRNAs and cancer: Biomarkers and master regulators. Semin Cancer Biol. 2019; 58: 90-99.

10. Lei M, Zheng G, Ning Q, Zheng J, Dong D. Translation and functional roles of circular RNAs in human cancer. Molecular Cancer. 2020; 19(1): 30.

11. Liu Z, Zhou Y, Liang G, Ling Y, Tan W, Tan L, et al. Circular RNA hsa_circ 001783 regulates breast cancer progression via sponging miR-200c-3p. Cell Death Dis. 2019; 10: 55

12. Zeng $\mathrm{K}, \mathrm{He} \mathrm{B}$, Yang BB, Xu T, Chen $\mathrm{X}, \mathrm{Xu} \mathrm{M}$, et al. The pro-metastasis effect of circANKS1B in breast cancer. Mol Cancer. 2018; 17: 160

13. Ye F, Gao G, Zou Y, Zheng S, Zhang L, Ou X, et al. circFBXW7 Inhibits Malignant Progression by Sponging miR-197-3p and Encoding a 185-aa Protein in Triple-Negative Breast Cancer. Molecular therapy Nucleic acids. 2019; 18: 88-98.

14. Xu X, Zhang J, Tian Y, Gao Y, Dong X, Chen W, et al. CircRNA inhibits DNA damage repair by interacting with host gene. Mol Cancer. 2020; 19: 128

15. Pan Y, Ma P, Liu Y, Li W, Shu Y. Multiple functions of m(6)A RNA methylation in cancer. J Hematol Oncol. 2018; 11: 48.

16. Liu J, Harada BT, He C. Regulation of Gene Expression by N(6)-methyladenosine in Cancer. Trends Cell Biol. 2019; 29(6): 487-499.

17. Wang S, Chai P, Jia R, Jia R. Novel insights on m(6)A RNA methylation in tumorigenesis: a double-edged sword. Mol Cancer. 2018; 17: 101.

18. Niu Y, Lin Z, Wan A, Chen H, Liang H, Sun L, et al. RNA N6-methyladenosine demethylase FTO promotes breast tumor progression through inhibiting BNIP3. Mol Cancer. 2019; 18(1):46.

19. Zhang C, Samanta D, Lu H, Bullen JW, Zhang H, Chen I, et al. Hypoxia induces the breast cancer stem cell phenotype by HIF-dependent and ALKBH5-mediated $\mathrm{m}^{6} \mathrm{~A}$-demethylation of NANOG mRNA. Proc Natl Acad Sci U S A. 2016; 113: E2047-56.

20. Cai X, Wang X, Cao C, Gao Y, Zhang S, Yang Z, et al. HBXIP-elevated methyltransferase METTL3 promotes the progression of breast cancer via inhibiting tumor suppressor let-7g. Cancer Lett. 2018; 415: 11-9.

21. Qian JY, Gao J, Sun X, Cao MD, Shi L, Xia TS, et al. KIAA1429 acts as an oncogenic factor in breast cancer by regulating CDK1 in an N6-methyladenosine-independent manner. Oncogene. 2019; 38: 6123-41.

22. Sun T, Wu Z, Wang X, Wang Y, Hu X, Qin W, et al. LNC942 promoting METTL14-mediated $\mathrm{m}(6) \mathrm{A}$ methylation in breast cancer cell proliferation and progression. Oncogene. 2020; 39(31): 5358-5372

23. Liu X, Gonzalez G, Dai X, Miao W, Yuan J, Huang M, et al. Adenylate Kinase 4 Modulates the Resistance of Breast Cancer Cells to Tamoxifen through an m(6)A-Based Epitranscriptomic Mechanism. Mol Ther. 2020; 28: 2593-2604.

24. Shi Y, Zheng C, Jin Y, Bao B, Wang D, Hou K, et al. Reduced Expression of METTL3 Promotes Metastasis of Triple-Negative Breast Cancer by m6A Methylation-Mediated COL3A1 Up-Regulation. Front Oncol. 2020; 10: 1126.

25. Wang H, Xu B, Shi J. N6-methyladenosine METTL3 promotes the breast cancer progression via targeting Bcl-2. Gene. 2020; 722: 144076.

26. Wang M, Yang Y, Yang J, Yang J, Han S. circ_KIAA1429 accelerates hepatocellular carcinoma advancement through the mechanism of m(6)A-YTHDF3-Zeb1. Life Sci. 2020; 257: 118082.

27. Di Timoteo G, Dattilo D, Centron-Broco A, Colantoni A, Guarnacci M, Rossi F, et al. Modulation of circRNA Metabolism by m(6)A Modification. Cell Rep. 2020; 31: 107641

28. Heo J, Noh BJ, Lee S, Lee HY, Kim Y, Lim J, et al. Phosphorylation of TFCP2L1 by CDK1 is required for stem cell pluripotency and bladder carcinogenesis. EMBO Mol Med. 2020; 12: e10880.

29. Ravindran Menon D, Luo Y, Arcaroli JJ, Liu S, KrishnanKutty LN, Osborne DG, et al. CDK1 Interacts with Sox2 and Promotes Tumor Initiation in Human Melanoma. Cancer Res. 2018; 78: 6561-6574.

30. Xi PW, Zhang X, Zhu L, Dai XY, Cheng L, Hu Y, et al. Oncogenic action of the exosome cofactor RBM7 by stabilization of CDK1 mRNA in breast cancer. NPJ Breast Cancer. 2020; 6: 58.

31. Kim SJ, Nakayama S Fau - Miyoshi Y, Miyoshi Y Fau - Taguchi T, Taguchi T Fau - Tamaki Y, Tamaki Y Fau - Matsushima T, Matsushima T Fau - Torikoshi $\mathrm{Y}$, et al. Determination of the specific activity of CDK1 and CDK2 as a novel prognostic indicator for early breast cancer. Ann Oncol. 2008; 19: 68-72.

32. Salmena L, Poliseno L Fau - Tay Y, Tay Y Fau - Kats L, Kats L Fau - Pandolfi PP, Pandolfi PP. A ceRNA hypothesis: the Rosetta Stone of a hidden RNA language? Cell. 2011; 146: 353-8.

33. Li X, Yang L, Chen LL. The Biogenesis, Functions, and Challenges of Circular RNAs. Mol Cell. 2018; 71: 428-42.

34. Liang $Y$, Song $X, \mathrm{Li} Y, \mathrm{Ma} T$, Su $\mathrm{P}$, Guo R, et al. Targeting the circBMPR2/ miR-553/USP4 Axis as a Potent Therapeutic Approach for Breast Cancer. Mol Ther Nucleic Acids. 2019; 17: 347-61.

35. Chen RX, Chen X, Xia LP, Zhang JX, Pan ZZ, Ma XD, et al $\mathrm{N}(6)$-methyladenosine modification of circNSUN2 facilitates cytoplasmic export and stabilizes HMGA2 to promote colorectal liver metastasis. Nat Commun. 2019; 10: 4695 
36. Zhang M, Huang N, Yang X, Luo J, Yan S, Xiao F, et al. A novel protein encoded by the circular form of the SHPRH gene suppresses glioma tumorigenesis. Oncogene. 2018; 37: 1805-14.

37. Pan Z, Cai J, Lin J, Zhou H, Peng J, Liang J, et al. A novel protein encoded by circFNDC3B inhibits tumor progression and EMT through regulating Snail in colon cancer. Mol Cancer. 2020; 19: 71

38. Song $X$, Liang $Y$, Sang $Y, \operatorname{Li} Y$, Zhang $H$, Chen B, et al. circHMCU Promotes Proliferation and Metastasis of Breast Cancer by Sponging the let-7 Family. Mol Ther Nucleic Acids. 2020; 20: 518-33.

39. He R, Liu P, Xie X, Zhou Y, Liao Q, Xiong W, et al. circGFRA1 and GFRA1 act as ceRNAs in triple negative breast cancer by regulating miR-34a. J Exp Clin Cancer Res. 2017; 36: 145 .

40. Tang H, Huang X, Wang J, Yang L, Kong Y, Gao G, et al. circKIF4A acts as a prognostic factor and mediator to regulate the progression of triple-negative breast cancer. Molecular Cancer. 2019; 18: 23.

41. Kong Y, Yang L, Wei W, Lyu N, Zou Y, Gao G, et al. CircPLK1 sponges miR-296-5p to facilitate triple-negative breast cancer progression. Epigenomics. 2019; 11: 1163-1176.

42. Han J, Wang JZ, Yang X, Yu H, Zhou R, Lu HC, et al. METTL3 promote tumor proliferation of bladder cancer by accelerating pri-miR221/222 maturation in m6A-dependent manner. Mol Cancer. 2019; 18: 110.

43. Wang Q, Chen C, Ding Q, Zhao Y, Wang Z, Chen J, et al. METTL3-mediated $\mathrm{m}(6) \mathrm{A}$ modification of HDGF mRNA promotes gastric cancer progression and has prognostic significance. Gut. 2020; 69: 1193-1205.

44. Lan T, Li H, Zhang D, Xu L, Liu H, Hao X, et al. KIAA1429 contributes to liver cancer progression through N6-methyladenosine-dependent posttranscriptional modification of GATA3. Mol Cancer. 2019; 18: 186. 